Cahiers de la recherche sur les droits

Cahiers

Fur les Droits fondamentaux

11 | 2013

Le droit de la famille en (r)évolutions

\title{
Le couple face au droit et à la procédure pénale : contribution à la mise en lumière d'un droit pénal du couple
}

Agnès Cerf-Hollender

\section{OpenEdition}

Journals

Édition électronique

URL : https://journals.openedition.org/crdf/4640

DOI : $10.4000 /$ crdf. 4640

ISSN : 2264-1246

Éditeur

Presses universitaires de Caen

Édition imprimée

Date de publication : 1 novembre 2013

Pagination : 45-54

ISSN : $1634-8842$

Référence électronique

Agnès Cerf-Hollender, « Le couple face au droit et à la procédure pénale : contribution à la mise en lumière d'un droit pénal du couple », Cahiers de la recherche sur les droits fondamentaux [En ligne], 11 | 2013, mis en ligne le 01 décembre 2014, consulté le 14 novembre 2022. URL : http:// journals.openedition.org/crdf/4640; DOI : https://doi.org/10.4000/crdf.4640 


\title{
Le couple face au droit et à la procédure pénale; contribution à la mise en lumière d'un droit pénal du couple
}

\author{
Agnès CERF-HOLLENDER \\ Maître de conférences en droit privé à I'Université de Caen Basse-Normandie \\ Centre de recherche sur les droits fondamentaux et les évolutions du droit (CRDFED, EA 2132)
}

I. La protection pénale du couple, déclinaison du droit pénal de la famille
A. La protection du couple par l'exclusion des règles pénales
$B$. La protection du couple par le renforcement des règles pénales

II. La protection pénale au sein du couple, déclinaison du droit pénal des personnes
A. La protection des personnes par l'aggravation de la répression
B. La protection des personnes par la séparation du couple

Le juriste cherche en vain dans les textes de loi une définition générale du couple, et doit donc se tourner vers son sens commun. Le mot vient du latin copula, qui signifie «lien ${ }^{1}$. Ce lien unissant deux personnes est fondé, sociologiquement, sur un subtil mélange de sentiments, de relations sexuelles, de communauté de vie, de biens et d'intérêts. Si le législateur ne le définit pas, il ne l'ignore pas pour autant, bien au contraire. Trois modalités de vie conjugale sont aujourd'hui reconnues par le Code civil: le classique et solennel mariage, le concubinage, simple union de fait, et depuis la loi du 15 novembre 1999, le pacte civil de solidarité (pacs), souvent présenté comme un intermédiaire des deux précédentes. L'approche civiliste du couple est ainsi aujourd'hui plurielle, car le droit de la famille, dans lequel il s'insère classiquement, a connu, ces dernières années, de profondes évolutions. Tout d'abord, si le couple demeure la cellule de base de la famille, il s'en détache aussi, de par la reconnaissance d'unions qui n'ont pas pour finalité de fonder une famille, mais simplement de vivre ensemble, afin de rompre la solitude, grand mal de notre siècle, et de partager sereinement une vie commune, les joies et les peines de chaque jour, et plus si affinité. Ensuite, le couple ne doit pas faire oublier les individus qui le composent. Comme l'a écrit le doyen Carbonnier, «la famille devient moins une institution qui vaudrait pour elle-même qu'un instrument offert pour l'épanouissement de sa personnalité ${ }^{2}$. Règne aujourd'hui dans le couple l'égalité, l'autonomie personnelle et un devoir de respect mutuel, inscrit dans le

1. A. Dauzat, Dictionnaire étymologique, Paris, Larousse, 1938.

2. J. Carbonnier, Essais sur les lois, $2^{\mathrm{e}}$ éd., Paris, Répertoire du notariat Defrénois, 1995, p. 171. 
Code civil par la loi du 4 avril $2006^{3}$. Tout cela a conduit la doctrine civiliste à mettre en lumière l'émergence d'un droit du couple, ou des couples ${ }^{4}$.

En matière pénale, le code de 1810 consacrait essentiellement, en ce qui concerne le couple, un ordre public de direction, tendant à assurer le respect de l'institution du mariage et des obligations en découlant: incrimination de la bigamie ${ }^{5}$, de la célébration d'un mariage religieux avant le mariage civil $^{6}$, de l'adultère ${ }^{7}$, puis, avec la loi du 7 février 1924, de l'abandon pécuniaire de famille ${ }^{8}$. Mais le droit pénal était réticent à s'immiscer dans les relations conjugales. Cela explique, notamment, les diverses immunités familiales, patrimoniales et extrapatrimoniales ${ }^{9}$, ainsi que, du côté de la jurisprudence, le refus d'admettre le viol entre époux, position aujourd'hui révolue ${ }^{10}$. La législation a ensuite beaucoup évolué. Le Code pénal de 1992 s'est voulu plus expressif des valeurs de notre temps, en particulier des droits de l'homme, et il s'est recentré sur la protection des personnes ${ }^{11}$. Une nouvelle circonstance aggravante, tenant à la qualité de conjoint ou de concubin de l'auteur de l'infraction a été créée ${ }^{12}$. Le coup d'envoi était donné au développement d'un ordre public de protection au sein du couple, qui se traduira ensuite, notamment, par plusieurs lois tendant à lutter contre les violences conjugales ${ }^{13}$.

Les textes pénaux renvoyant au "conjoint», «concubin», "partenaire lié par un pacte civil de solidarité», ou plus vaguement à la «personne vivant maritalement» sont aujourd'hui nombreux. Il arrive que cette référence au couple s'inscrive dans le cadre plus large de la famille, au même titre que les ascendants ou les descendants, voire les alliés, mais ce n'est pas toujours le cas. L'autonomie et la diversité du droit pénal doivent ici être soulignées. Tantôt les trois modalités civiles de vie conjugale sont mises sur un pied d'égalité, tantôt seuls le mariage et le pacs sont pris en compte, tantôt encore, seul le mariage a une incidence pénale, et dans ce dernier cas, la loi exige parfois que les époux ne soient pas séparés de corps, autorisés à résider séparément, ou aient une vie commune effective, ou encore que l'union ait duré un certain nombre d'années. Mais le droit pénal va encore plus loin en prenant parfois en considération des couples qui ne sont pas encore formés, et qui ne le seront peut-être - sans doute - jamais, ou encore des couples qui n'existent plus, et échappent totalement au droit civil. De plus, en feuilletant les codes, on ne peut que remarquer un éparpillement des dispositions relatives au couple. Comme nous le verrons, le couple peut avoir des incidences en matière d'atteintes aux personnes, d'infractions contre les biens, contre l'État, de peines et de droit pénitentiaire, de procédure. Émerge ainsi, comme en droit civil, un droit pénal du couple ${ }^{14}$.

La présente étude tentera de contribuer modestement à la mise en lumière de ce droit pénal du couple composé de dispositions éparses et variées. L'ordre public de direction n'a pas disparu, comme en atteste l'incrimination des mariages naturalisants ${ }^{15}$ ou les dispositions relatives au mariage forcé ${ }^{16}$, mais le législateur se veut aujourd'hui avant tout doublement protecteur: protecteur du couple lui-même (I), mais aussi, en son sein, protecteur de chacun de ses membres (II). Dans la première hypothèse, le droit pénal du couple constitue une déclinaison du droit pénal de la famille, dans la seconde, une déclinaison du droit pénal des personnes.

3. Code civil, art. 212, loi nº 2006-399 du 4 avril 2006 renforçant la prévention et la répression des violences au sein du couple ou commises contre les mineurs. Voir, notamment, M. Avazant, «Regard civiliste sur la loi du 4 avril 2006 », Droit de la famille, 2006, étude n 40 ; P. Bonfils, E. Vergès, Chronique législative, Revue de sciences criminelles, 2007, p. 337-343; A.-M. Leroyer, "Regard civiliste sur la loi relative aux violences au sein du couple", Revue trimestrielle de droit civil, 2006, p. 402-410; M. Lobe-Lobas, «La prévention et la répression des violences commises au sein du couple ou contre les mineurs », Les petites affiches, $n^{\circ}$ 139, 13 juillet 2006, p. 5-10; P. Murat, "Proposition de loi sur les violences intrafamiliales: beaucoup de symboles pour quelle efficacité?», Droit de la famille, 2006, repère $\mathrm{n}^{\circ} 1$; M. Rebourg, "Prévention et répression des violences au sein du couple ou commises contre les mineurs", La semaine juridique, éd. G, 2006, actualité nº 173; D. Viriot-Barrial, "Commentaire de la loi du 4 avril 2006», Dalloz, 2006, Chron., p. 2350-2356.

4. Voir C. Brunetti-Pons, «L'émergence d'une notion de couple en droit civil», Revue trimestrielle de droit civil, 1999, p. 27; J.-J. Lemouland, "L'émergence d'un droit des couples», in Mariage-conjugalité, parenté-parentalité, H. Fulchiron (dir.), Paris, Dalloz (Thèmes et commentaires), 2009, p. 33. Voir aussi J.-F. Sagaut, «Le couple conjugal complément ou substitut à la solidarité intergénérationnelle? », Droit de la famille, 2011, dossier n 11, "Couples, patrimoine: les défis de la vie à deux", 106 Congrès des notaires de France, Bordeaux, juin 2010 ".

5. Code pénal 1810, art. 340; Code pénal 1992, art. 433-20.

6. Code pénal 1810, art. 199 et 200 ; Code pénal 1992, art. 433-21.

7. Code pénal 1810, art. 336 à 339, abrogés par la loi du 11 juillet 1975 sur le divorce.

8. Code pénal 1810, art. 357-2; Code pénal 1992, art. 227-3.

9. Voir infra, $1^{\text {re }}$ partie.

10. Aux motifs que le mariage engendre une présomption de consentement à l'acte sexuel: Cass., crim., 19 mars 1910, Bulletin criminel, nº 153 . Cette approche a été abandonnée en 1992 : voir infra, $2^{\mathrm{e}}$ partie.

11. Voir Le nouveau code pénal, enjeux et perspectives (Actes du colloque organisé par le ministère de la Justice, Paris, 1994), P. Méhaignerie (dir.), Paris, Dalloz (Thèmes et commentaires), 1994.

12. Code pénal, art. 132-80; voir infra, II, A.

13. Loi no 2006-399 du 4 avril 2006, précisément, et loi no 2010-769 du 9 juillet 2010, relative aux violences spécifiquement faites aux femmes, aux violences au sein du couple et aux incidences de ces dernières sur les enfants. Voir, notamment, I. Corpart, «Intensification de la lutte contre les violences conjugales", Droit de la famille, 2010, étude n 28 ; F. Dekeuwer-Defossez, "Les aspects civils de la loi relative aux violences faites spécifiquement aux femmes", Revue Lamy droit civil, octobre 2010, p. 43; C. Pomart-Nomdédéo, "Droit pénal et droit de la famille, les liaisons dangereuses ", Droit de la famille, 2010, étude n 20; A.-G. Robert, Chronique législative, Revue de sciences criminelles, 2010, p. 911-922.

14. Voir P. Maistre du Chambon, "Quelques considérations sur le droit pénal de la famille», La semaine juridique, éd. G, Libres propos, $2011,6$.

15. CESEDA, art. L. 623-1. Voir aussi Sénat, Proposition de loi n ${ }^{\circ} 501$ du 26 mai 2010 visant à créer un délit de polygamie et d'incitation à la polygamie: M. Lamarche, «Ne confondons pas bigamie et polygamie», Droit de la famille, 2010, alerte $n^{\circ} 47$.

16. Voir infra, II, A. 


\section{La protection pénale du couple, déclinaison du droit pénal de la famille}

La protection pénale du couple est assurée par des dispositions très variées, et même, pourrait-on dire, disparates. Disparates tout d'abord car le couple protégé est entendu tantôt très largement, incluant les époux (y compris après un divorce), les partenaires et les concubins, tantôt très restrictivement, se limitant au mariage, et exigeant parfois, en outre, une vie commune effective. Disparates ensuite car la protection pénale se traduit soit par une exclusion de l'application de certaines règles $(\mathrm{A})$, exclusion destinée à garantir la solidarité et les liens familiaux, soit par un renforcement des règles pénales (B), afin de maintenir le lien conjugal ou de sanctionner les atteintes qui pourraient être portées aux personnes, en raison de leur situation conjugale.

\section{A. La protection du couple par l'exclusion des règles pénales}

La protection par exclusion des règles pénales connaît trois illustrations: les immunités familiales, la dispense de témoigner en justice et l'interdiction de prononcer certaines peines.

Les immunités familiales, qui peuvent être d'ordre patrimonial ou extrapatrimonial, ont pour effet d'exclure les membres d'une même famille du champ pénal, soit parce qu'elles sont un obstacle à l'action publique, soit parce qu'elles constituent une cause d'irresponsabilité ${ }^{17}$. Elles sont anciennes et leurs fondements ont été de tout temps controversés, du moins en ce qui concerne les premières ${ }^{18}$. Il semble toutefois acquis aujourd'hui qu'elles ont toutes deux un fondement social, visant « à assurer la protection de la famille, élément naturel et fondamental de la société $[. .$.$] dont l'harmonie doit être préservée { }^{19}$. Si elles concernent la «famille», il n'en demeure pas moins, et c'est ce que nous allons montrer ci-après, qu'elles se sont petit à petit recentrées sur le couple, soit par la limitation de leur champ, pour les immunités patrimoniales, soit par son extension, pour les immunités extrapatrimoniales.

Les immunités d'ordre patrimonial s'appliquent aux cinq principales infractions contre les biens: le vol, l'extorsion, le chantage, l'escroquerie et l'abus de confiance ${ }^{20}$. L'article 380 de l'ancien Code pénal incluait dans ses bénéficiaires les alliés au même degré, à condition que l'infraction soit commise pendant le mariage et que les époux ne soient pas autorisés à résider séparément. Quant aux époux, l'immunité jouait largement, pendant le mariage, sans condition de vie commune, et perdurait au-delà, au profit du veuf ou de la veuve. Le Code pénal de 1992 a limité son champ : les alliés en sont désormais exclus, l'immunité ne jouant plus que pour les infractions commises au préjudice « de son ascendant ou de son descendant» $\left(1^{\circ}\right)$ et "de son conjoint, sauf lorsque les époux sont séparés de corps ou autorisés à résider séparément» $\left(2^{\circ}\right)$. Exit donc le veuf et la veuve. De plus, la loi du 4 avril 2006 a exclu le jeu de l'immunité lorsque l'infraction porte sur « des objets ou documents indispensables à la vie quotidienne de la victime, tels que des documents d'identité, relatifs au titre de séjour ou de résidence d'un étranger, ou des moyens de paiement ${ }^{21}$. L'immunité s'est ainsi recentrée sur le couple, mais pas n'importe lequel: le couple marié et de surcroît uni par une vie commune (quoique l'immunité ne soit pas écartée dans le cas d'une séparation de fait) et par le respect mutuel, la réserve posée par la loi du 4 avril 2006 tendant à éviter de maintenir un époux sous la dépendance de l'autre. Se trouvent ainsi combinées la protection du couple et celle de ses membres.

Les immunités extrapatrimoniales s'appliquent à certaines entraves à la saisine de la justice prévues par le livre 4 du Code pénal: délits de non-dénonciation de crime $^{22}$, de recel de criminel ou de terroriste ${ }^{23}$, de défaut de témoignage en faveur d'un innocent ${ }^{24}$. Dans le Code pénal de 1810, bénéficiaient de l'immunité «les parents ou alliés, jusqu'au quatrième degré inclusivement, des auteurs ou complices du crime ${ }^{25}$. Le conjoint n'était pas expressément visé, même si la doctrine considérait qu'il devait être inclus au titre d'allié ${ }^{26}$. Le Code pénal de 1992 a ici encore recentré l'immunité sur le lien de couple, et cela doublement. Bénéficient de l'immunité, d'une part, «le conjoint de l'auteur ou du complice, ou la personne qui vit notoirement en situation maritale avec lui ${ }^{27}$, formule large qui permet d'inclure les autres modalités de vie conjugale, et qui a évité au législateur de modifier les textes lors de la création du pacs; d'autre

17. Sur la distinction entre les «immunités-irrecevabilité» et les «immunités-irresponsabilité», voir P. Mousseron, «les immunités familiales», Revue de sciences criminelles, 1998, p. 291-300; Y. Mayaud, «Rebondissement sur les immunités familiales: fond ou forme?», in De code en code: mélanges en l'honneur du doyen Georges Wiederkehr, M. Puech (dir.), Paris, Dalloz, 2009, p. 541-550.

18. Pour un exposé de ces fondements, voir G. Clément, "L'immunité familiale d'ordre patrimonial», in Mélanges en l'honneur du professeur Jacques-Henri Robert, Paris, LexisNexis, 2012, p. 108-110.

19. G. Clément, «L'immunité familiale...», p. 110.

20. Code pénal, art. 311-12; 312-9, al. 2 ; 312-12, al. 2 ; 313-3, al. 2 et 314-4, al. 2.

21. Loi du 4 avril 2006, art. 9, codifié à l'art. 311-12, al. 2 du Code pénal.

22. Code pénal, art. 434-1. L'immunité est écartée pour les crimes commis sur mineur de quinze ans.

23. Code pénal, art. 434-6.

24. Code pénal, art. 434-11.

25. Code pénal 1810, art. 61, al. 3 (recel de criminel); art. 62, al. 3 (non-dénonciation de crime); art. 63, al. 4 (défaut de témoignage en faveur d'un innocent).

26. Voir R. Merle, A. Vitu, Traité de droit criminel. Droit pénal spécial, Paris, Cujas, 1981, t. 1, n 507, p. 385.

27. Code pénal, art. 434-1, al. $2,2^{\circ}$; art. $434-6$, al. 2, $2^{\circ}$; art. 434-11, al. $3,3^{\circ}$. 
part, «les parents en ligne directe et leurs conjoints, ainsi que les frères et sœurs et leurs conjoints, de l'auteur ou du complice ${ }^{28}$.

Est prévue aussi une immunité extrapatrimoniale qui concerne le délit d'aide à l'entrée et au séjour irrégulier d'un étranger de l'article L. 622-4 du CESEDA. Son évolution s'est elle aussi faite au profit du couple, mais avec des hauts et des bas, tant la législation sur les étrangers est mouvante. Initialement, l'article 21 III de l'ordonnance du 2 novembre 1945, issu de la loi no 96-647 du 22 juillet $1996^{29}$, visait comme seules personnes immunisées les ascendants ou descendants de l'étranger, ainsi que son conjoint, sauf lorsque les époux étaient séparés de corps ou autorisés à résider séparément. La loi no 98-349 du 11 mai $1998^{30}$ avait étendu son champ aux conjoints des ascendants, descendants, frères et sœurs de l'étranger, et à la personne vivant maritalement avec lui. Allant à contre-courant, la codification de l'ordonnance de 1945, devenue le CESEDA ${ }^{31}$, fut l'occasion d'exclure du bénéfice de l'immunité les conjoints des ascendants, descendants et frères et sœurs, si les époux étaient séparés de corps, avaient un domicile distinct ou étaient autorisés à résider séparément, de même que le conjoint de l'étranger, si les époux étaient séparés de corps, autorisés à résider séparément ou si la communauté de vie entre eux avait cessé. La loi no $2012-1560$ du 31 décembre $2012^{32}$ est revenue à une plus large prise en compte du couple en supprimant, en toute hypothèse, l'exigence de vie commune, et en étendant l'immunité "aux frères et sœurs du conjoint de l'étranger ou de la personne qui vit notoirement en situation maritale avec lui».

La deuxième illustration de la protection du couple par exclusion des règles pénales est la dispense de prêter serment accordée, pour motif familial, à certains proches du prévenu ou de l'accusé. Rappelons que tout témoin cité en justice a l'obligation de comparaître, de prêter serment de dire la vérité et de déposer, sous peine de sanction ${ }^{33}$. Toutefois, des exceptions à la prestation de serment sont prévues à l'égard des père et mère du prévenu ou de l'accusé, ainsi que de ses ascendants, descendants, de ses frères et sœurs, de ses alliés au même degré et de son conjoint, y compris après divorce ${ }^{34}$. Cette dispense peut être analysée comme complémentaire aux immunités extrapatrimoniales exposées ci-dessus : il est en effet cohérent que celui ou celle qui n'est pas tenu de dénoncer un proche ne soit pas tenu non plus de prêter serment de dire la vérité lorsqu'il est entendu au cours de son procès. Et pourtant, son champ, quant au couple, est beaucoup plus restreint et reste attaché à une conception traditionaliste de la famille: cette dispense est limitée au «conjoint», et la chambre criminelle refuse de l'étendre au concubin ${ }^{35}$ et au partenaire lié par un pacs ${ }^{36}$, avec l'aval de la Cour européenne des droits de l'homme ${ }^{37}$.

La dernière manifestation de la protection du couple par exclusion des règles pénales concerne les peines. Il est incontestable que la condamnation pénale d'une personne rejaillit sur celles et ceux qui partagent sa vie. Outre l'opprobre jeté sur la famille, certaines peines peuvent affecter directement le conjoint du condamné. Par exemple, l'emprisonnement ferme le privera de vie commune, les peines pécuniaires, telles l'amende ou la confiscation, affecteront le patrimoine familial, au premier chef le conjoint ${ }^{38}$. Toutefois, une seule peine est écartée pour motif de lien conjugal. Il s'agit de l'interdiction du territoire français pouvant être prononcée, à titre définitif ou pour une durée de dix ans au plus, à l'encontre de tout étranger coupable d'un crime ou d'un délit ${ }^{39}$. Afin de concilier cette peine avec le droit à une vie familiale posé par l'article 8 de la Convention européenne des droits de l'homme, l'article 131-30-2 du Code pénal interdit son prononcé dans certains cas ${ }^{40}$, en particulier à l'égard de l'étranger qui cumule les conditions suivantes: il réside régulièrement en France depuis plus de dix ans; il ne vit pas en état de polygamie; il est marié depuis au moins quatre ans ${ }^{41}$ avec un ressortissant français ayant conservé la nationalité française ou avec un ressortissant étranger

28. Code pénal, art. 434-1, al. 2, $1^{\circ}$ (nous soulignons). Ici, seul le mariage est pris en compte.

29. Loi tendant à renforcer la répression du terrorisme et des atteintes aux personnes dépositaires de l'autorité publique ou chargées d'une mission de service public et comportant des dispositions relatives à la police judiciaire, art. 25.

30. Loi relative à l'entrée et au séjour des étrangers en France et au droit d'asile, art. 12.

31. Ordonnance $n^{\circ}$ 2004-1248 du 24 novembre 2004, ratifiée par la loi nº 2006-911 du 24 juillet 2006. Par ailleurs, l'immunité a été exclue, et l'est toujours, si l'étranger vit en état de polygamie.

32. Loi relative à la retenue pour vérification du droit au séjour et modifiant le délit d'aide au séjour irrégulier pour en exclure les actions humanitaires et désintéressées, art. 12.

33. Code de procédure pénale, art. 326 pour la cour d'assises et art. 438 pour le tribunal correctionnel.

34. Code de procédure pénale, art. 335 pour la cour d'assises et art. 448 pour le tribunal correctionnel. Toutefois, leur audition sous serment n'entraîne pas la nullité lorsque le ministère public ni aucune des parties ne s'est opposé à la prestation de serment: Code de procédure pénale, art. 336 et 449 .

35. Cass., crim., 21 mars 1973, Bulletin criminel, $\mathrm{n}^{\circ} 141$

36. Cass., crim., 25 mai 2011, $\mathrm{n}^{\circ} 10-86229$, Bulletin criminel, $\mathrm{n}^{\circ} 109$.

37. Cour EDH, Van der Heijden c. Pays-Bas (GC), 3 avril 2012, req. $\mathrm{n}^{\circ} 42857 / 05$.

38. Outre les biens (meubles, immeubles, droits intellectuels, divis ou indivis) ayant servi à commettre l'infraction ou qui étaient destinés à la commettre, la confiscation peut aussi porter, pour les crimes et les délits punis de cinq ans au moins d'emprisonnement, «et ayant procuré un profit direct ou indirect», sur tous les biens «appartenant au condamné ou, sous réserve des droits du propriétaire de bonne foi, dont il a la libre disposition, lorsque ni le condamné, ni le propriétaire, mis en mesure de s'expliquer sur les biens dont la confiscation est envisagée, n'ont pu en justifier l'origine»; Code pénal, art. 131-21.

39. Code pénal, art. 131-30.

40. Les autres cas tiennent notamment à la durée de résidence en France et au fait d'être parent d'un enfant mineur résidant en France.

41. La durée du mariage, auparavant de trois ans, a été portée à quatre ans par la loi nº 2006-911 du 24 juillet 2006. 
qui réside en France habituellement depuis qu'il a atteint au plus l'âge de treize ans; le mariage est antérieur aux faits ayant entraîné sa condamnation; la communauté de vie n'a pas cessé depuis le mariage; les faits à l'origine de la condamnation n'ont pas été commis à l'encontre du conjoint ou des enfants de l'étranger. Ces multiples conditions reflètent toutes les attitudes du droit pénal face au couple: la protection, certes lourdement encadrée, du lien conjugal, mais aussi la protection du conjoint au sein du couple et un ordre public de direction (prohibition de la polygamie, absence d'effet du mariage postérieur à l'infraction qui aurait pour objet d'échapper à une reconduite à la frontière). Lorsque ces conditions ne sont pas remplies, le lien conjugal sera toutefois pris en compte, non par une exclusion de la peine, mais par un renforcement des conditions de son prononcé.

\section{B. La protection du couple par le renforcement des règles pénales}

Les règles pénales renforcées en raison de la situation conjugale peuvent être rangées en deux grandes catégories. La première se compose des dispositions tendant à maintenir les relations lorsque le couple se trouve séparé en raison de la condamnation ou de l'implication de l'un de ses membres dans une procédure pénale. La seconde comprend des dispositions tendant à prévenir ou sanctionner des infractions commises en raison du lien conjugal. Mais, comme nous allons le voir, le couple est généralement pris en compte de manière diffuse, au travers de la famille.

La première catégorie trouve illustration essentiellement en matière de peines. Revenons sur l'interdiction du territoire français. Comme cela a été vu précédemment, cette peine est parfois exclue. Mais si les conditions très strictes de son exclusion ne sont pas remplies, elle est cependant adaptée, pour le même motif de respect du droit à une vie familiale: son prononcé doit être, en matière correctionnelle, spécialement motivé lorsque le condamné est un «étranger marié depuis au moins trois ans $^{42}$ avec un conjoint de nationalité française, à condition que ce mariage soit antérieur aux faits ayant entraîné sa condamnation, que la communauté de vie n'ait pas cessé depuis le mariage et que le conjoint ait conservé la nationalité française ${ }^{43}$.
Le lien conjugal peut aussi avoir une incidence sur les modalités d'exécution des peines privatives de liberté, mais de manière plus diffuse. Ainsi, la semi-liberté, le placement sous surveillance électronique, peuvent être accordés au condamné qui justifie «de sa participation essentielle à la vie de sa famille» ${ }^{44}$. Le fractionnement de la peine peut être décidé pour des motifs d'ordre «familial» ${ }^{45}$. De même, la libération conditionnelle peut être octroyée aux condamnés qui justifient de leur «participation essentielle à la vie de leur famille» ${ }^{46}$. Certes, dans toutes ces hypothèses, le conjoint n'est pas spécialement visé, mais il est nécessairement inclus. Un désir identique de maintien des liens familiaux et conjugaux se retrouve lorsque le condamné est incarcéré, surtout depuis la loi pénitentiaire du 24 novembre $2009^{47}$. Sans entrer dans tous les détails, signalons seulement quelques mesures significatives: le juge d'instruction ne peut refuser un permis de visite à un membre de la famille du mis en examen détenu que par décision écrite spécialement motivée ${ }^{48}$; sont prévus, pour les visites, des

[...] parloirs familiaux [...] spécialement conçus afin de permettre aux personnes détenues de recevoir, sans surveillance continue et directe, des visites des membres majeurs de leur famille ou de proches majeurs accompagnés, le cas échéant, d'un ou de plusieurs enfants mineurs, pendant une durée de six heures au plus au cours de la partie diurne de la journée ${ }^{49}$,

ainsi que des «unités de vie familiale», locaux qui permettent, sous les mêmes conditions, de recevoir les mêmes personnes pendant une durée comprise entre six heures et soixante-douze heures ${ }^{50}$. Les textes sont là. Mais ne nous voilons pas la face: leur mise en œuvre se heurte à des obstacles financiers considérables, lorsque l'on sait les conditions déplorables de détention en France, régulièrement soulignées par le Contrôleur général des lieux de privations de liberté ${ }^{51}$.

Le Code de procédure pénale veille aussi aux liens familiaux en amont, au cours de l'enquête, et plus particulièrement de la garde à vue. La personne placée en garde à vue dispose du droit de faire prévenir par téléphone, notamment, « une personne avec laquelle elle vit habituellement ou l'un de ses parents en ligne directe, l'un de ses frères et sœurs ${ }^{52}$. De plus, si le gardé à vue ne sollicite pas d'examen médical, ce dernier peut être demandé par

42. La durée exigée du mariage a été régulièrement allongée: fixée à un an par la loi nº 93-1027 du 24 août 1993, elle est passée à deux ans (loi nº 2003 1119 du 26 novembre 2003), puis à trois ans (loi n² 2006-911 du 24 juillet 2006).

43. Code pénal, art. 131-30-1, $2^{\circ}$.

44. Code pénal, art. 132-25, $2^{\circ}$; art. 132-26-1. La peine prononcée ou restant à subir doit être égale ou inférieure à deux ans, ou à un an si le condamné est en état de récidive.

45. Code pénal, art. 132-27.

46. Code de procédure pénale, art. 729 , al. $2,2^{\circ}$.

47. Loi nº 2009-1436. Voir J.-P. Céré, «La loi du 24 novembre 2009: aspects de procédure pénale et de droit pénitentiaire », Droit pénal, 2010, étude n 2.

48. Code de procédure pénale, art. 145-4.

49. Code de procédure pénale, art. R. 57-8-13.

50. Code de procédure pénale, art. R. 57-8-14

51. Barbara Gonçalves, «Rapport d'activité 2012 et bilan contrasté pour le Contrôleur général des lieux de privation de liberté», Lettre "Actualités Droits-Libertés » du CREDOF, 5 mars 2013.

52. Code de procédure pénale, art. 63-2. 
un «membre de sa famille» ${ }^{53}$. Certes, ici encore, le lien de couple n'est pas expressément visé, mais il se retrouve en filigrane.

La seconde catégorie de règles protectrices tend à prévenir ou sanctionner des infractions commises en raison du lien conjugal. La première disposition qui vient à l'esprit est le délit de discrimination, dont l'un des motifs peut tenir à la «situation de famille» de la victime ${ }^{54}$. Nous insisterons plutôt sur une hypothèse moins connue: celle dans laquelle la loi étend la protection accordée à une personne déterminée, en raison de sa qualité ou de ses fonctions, à ses proches. Le conjoint (et parfois le partenaire et le concubin) va être protégé "par ricochet», en tant que victime collatérale effective ou potentielle. Cela se rencontre tout d'abord au profit de personnes bénéficiant, au cours d'une procédure pénale, de l'anonymat, ou autorisées à user d'une identité d'emprunt. Ainsi, le juge des libertés et de la détention peut autoriser un témoignage anonyme lorsque l'audition est susceptible de mettre gravement en danger la vie ou l'intégrité physique du témoin, mais aussi «des membres de sa famille ou de ses proches $"{ }^{55}$. La protection est encore plus importante à l'égard des repentis ${ }^{56}$ ou des agents infiltrés ${ }^{57}$ : le fait de révéler leur identité d'emprunt (pour les repentis) ou réelle (pour les agents infiltrés) est puni de cinq ans d'emprisonnement et de 75000 euros d'amende, ces peines passant à sept ans d'emprisonnement et à 100 ooo euros d'amende lorsque cette révélation a causé, directement ou indirectement, des violences, coups et blessures à leur encontre ou à celle «de leurs conjoints, enfants et ascendants directs », et à dix ans d'emprisonnement et à 150000 euros d'amende lorsqu'elle a causé, directement ou indirectement, la mort de ces mêmes personnes. Il est étonnant que la loi ne vise que le conjoint, d'autant plus que la protection similaire accordée à une autre catégorie d'agents, les agents des services spécialisés de renseignement, s'étend non seulement à leur conjoint, mais aussi à leur partenaire lié par un pacs ${ }^{58}$.

Enfin, on retrouve une telle protection par ricochet dans le Code pénal. Certaines circonstances aggravantes des atteintes volontaires à la vie ou à l'intégrité physique sont liées aux fonctions ou à la profession de la victime: magistrat, juré, avocat, officier public ou ministériel, militaire de la gendarmerie nationale, fonctionnaire de la police nationale, des douanes, de l'administration pénitentiaire ou toute autre personne dépositaire de l'autorité publique, sapeur-pompier professionnel ou volontaire, gardien assermenté d'immeubles, agent exerçant pour le compte d'un bailleur des fonctions de gardiennage ou de surveillance d'immeubles à usage d'habitation, enseignant, personnel travaillant dans les établissements d'enseignement scolaire, agent d'un exploitant de réseau de transport public de voyageurs ou toute personne chargée d'une mission de service public, professionnel de santé ${ }^{59}$. La circonstance aggravante joue aussi lorsque l'infraction est commise, en raison des fonctions exercées par ces personnes, non pas sur elles-mêmes, mais sur leur « conjoint, ascendants, descendants en ligne directe» ou «sur toute autre personne vivant habituellement» à leur domicile ${ }^{60}$, ce qui permet d'inclure les concubins et partenaires liés par un pacs. Un mécanisme identique est prévu pour le délit de destruction ou dégradation volontaire de biens ${ }^{61}$.

Ainsi, le droit pénal assure au couple, en tant que cellule de base de la famille, une protection, souvent différenciée en fonction des modalités de vie conjugale, qui va parfois jusqu'à le mettre hors la loi pénale. Tout autre est la situation dans laquelle une infraction est commise au sein du couple. La protection pénale s'accentue et se déplace alors au profit des victimes individuelles.

\section{La protection pénale au sein du couple, déclinaison du droit pénal des personnes}

La protection pénale au sein du couple s'inscrit aujourd'hui dans le cadre, plus large, de la lutte contre les violences conjugales. Le champ pénal s'avère alors particulièrement large. D'une part, tous les couples sont mis sur un pied d'égalité, sans distinction, d'autre part, le droit pénal dépasse les bornes temporelles tant du mariage que du pacs ou du concubinage, pouvant intervenir en amont, avant la formation de l'union (notamment dans le cadre de la lutte contre les mariages forcés), et en aval, après un divorce ou une séparation, sans limitation de durée et même si plus aucune relation de nature civile ne subsiste. La protection se traduit par une aggravation et une autonomisation de la répression (A) et par la faculté de décider la séparation du couple (B).

53. Code de procédure pénale, art. 63-3, al. 3. L'examen médical est de droit. L'équivalent n'est en revanche pas prévu pour la mise en œuvre du droit à l'assistance d'un avocat.

54. Code pénal, art. 225-1.

55. Code de procédure pénale, art. 706-58. La révélation de l'identité ou de l'adresse du témoin est punie de cinq ans d'emprisonnement et 75 ooo € d'amende (Code de procédure pénale, art. 706-59, al. 2).

56. Code de procédure pénale, art. 706-63-1.

57. Code de procédure pénale, art. 706-84.

58. Code pénal, art. 413-13.

59. Code pénal, art. 221-4, $4^{\circ}$ et $4^{\circ}$ bis (meurtre); art. 222-3, $4^{\circ}$ et $4^{\circ}$ bis (actes de tortures et de barbarie); art. 222-8, 222-10, 222-12, 222-13, $4^{\circ}$ et $4^{\circ}$ bis (violences de toute gravité).

60. Mêmes articles, circonstances aggravantes prévue par le $4^{\circ}$ ter.

61. Code pénal, art. $322-3,3^{\circ}$ bis. 


\section{A. La protection des personnes par l'aggravation de la répression}

La première étape marquante de l'aggravation de la répression fut l'introduction, par le Code pénal de 1992, d'une circonstance aggravante nouvelle, tenant à la qualité de conjoint ou de concubin de l'auteur de l'infraction. À l'origine, elle figurait seulement dans la partie spéciale du Code pénal, attachée aux infractions auxquelles elle s'appliquait, c'est-à-dire les actes de torture et de barbarie et les violences volontaires de toute gravité ${ }^{62}$. La loi no $2006-399$ du 4 avril 2006 lui a donné une place plus symbolique, qui se doit d'être soulignée. Elle l'a insérée dans le livre $1^{\text {er }}$ du Code, consacré aux dispositions générales ${ }^{63}$, alors que d'autres circonstances aggravantes de nature familiale (on pense notamment à la qualité d'ascendant ou de descendant) sont toujours posées seulement dans la partie spéciale.

La loi de 2006 a aussi largement étendu le champ de cette circonstance aggravante. Tout d'abord, elle a inclus, aux côtés du conjoint et du concubin, le partenaire lié par un pacs, extension indispensable pour éviter une disparité injustifiée entre les trois modalités civiles de vie conjugale. Ensuite, elle a prévu que la circonstance perdure après le divorce ou la rupture de la relation, car nul ne conteste que la séparation ne met pas toujours fin aux tensions au sein du couple, qui peuvent même s'en trouver exacerbées ${ }^{64}$. Aucune limite dans le temps n'est posée: la loi exige seulement un lien de causalité entre l'infraction et les relations passées entre l'auteur et la victime, ce qui n'est pas facile à établir ${ }^{65}$. À la différence du lien civil, qui peut être rompu, le lien pénal est devenu perpétuel. Enfin, la loi a allongé la liste des infractions aggravées, ajoutant aux violences le meurtre et les agressions sexuelles ${ }^{66}$. Pour ces dernières, elle a clairement détaché le droit pénal du couple du «devoir conjugal» du droit civil, en énonçant que :

[1]e viol et les autres agressions sexuelles sont constitués [...] quelle que soit la nature des relations existant entre l'agresseur et sa victime, y compris s'ils sont unis par les liens du mariage [...] la présomption de consentement des époux à l'acte sexuel ne vaut que jusqu'à preuve contraire ${ }^{67}$.

Elle a ainsi consacré la jurisprudence interne et européenne reconnaissant le viol entre époux ${ }^{68}$. Il n'en demeure pas moins que le refus d'avoir des relations sexuelles avec son conjoint reste une faute civile cause de divorce, source de dommages et intérêts ${ }^{69}$ et que la preuve contraire sera plus difficile à apporter en raison du lien matrimonial ${ }^{70}$.

La loi no 2010-769 du 9 juillet 2010 a franchi un nouveau cap en autonomisant le droit pénal du couple. Elle a créé une qualification spécifique aux menaces commises par le conjoint, le concubin ou le partenaire ${ }^{71}$, et deux nouvelles incriminations pour lesquelles le lien conjugal, présent ou passé, entre l'auteur et la victime n'est plus seulement une circonstance aggravante, mais un élément constitutif de l'infraction. La première est caractérisée par des «violences habituelles» commises par le conjoint, le concubin ou le partenaire (ou «ex») de la victime, inspirée des violences habituelles sur mineur de quinze ans ou personne vulnérable ${ }^{72}$. Elle constitue un crime lorsque les violences ont entraîné la mort, une mutilation ou une infirmité ${ }^{73}$, et un délit dans les autres cas ${ }^{74}$. La seconde est le harcèlement conjugal, défini comme «le fait de harceler son conjoint, son partenaire lié par un pacte civil de solidarité ou son concubin par des agissements répétés ayant pour objet ou pour effet une dégradation de ses conditions de vie se traduisant par une altération de sa santé physique ou mentale ${ }^{75}$. Inspirée du harcèlement

62. Code pénal, art. 222-3, $6^{\circ}$ (actes de torture et de barbarie); art. 222-8, $6^{\circ}$ (violences volontaires ayant entraîné la mort); art. 222-10, $6^{\circ}$ (violences volontaires ayant entraîné mutilation ou infirmité); art. 222-12, $6^{\circ}$ (violences volontaires ayant entraîné plus de huit jours d'incapacité totale de travail); art. 222-13, $6^{\circ}$ (violences volontaires ayant entraîné moins de huit jours d'incapacité de travail ou aucune incapacité).

63. Code pénal, art. 132-80. La circonstance aggravante reste spéciale, ne s'appliquant qu'aux infractions pour lesquelles elle est expressément prévue.

64. Code pénal, art. 132-80, al. 2. Voir M.-F. Vievielle-Miravette, «La circonstance aggravante d'ex en matière de violences au sein du couple», Droit pénal, 2009, étude $\mathrm{n}^{\circ} 4$.

65. Cass., crim., 7 avril 2009, nº8-87.480, Droit pénal, 2009, comm. 93, obs. M. Véron (violences commises lors de la remise des enfants au prévenu, alors que leur mère se plaignait du retard dans le paiement de la pension alimentaire).

66. Code pénal, art. 221-4, $9^{\circ}$ (meurtre); art. 222-24, $11^{\circ}$ (viol); art. 222-28, $7^{\circ}$ (autres agressions sexuelles).

67. Code pénal, art. 222-22, al. 2. La référence à une présomption de consentement à l'acte sexuel a été supprimée par la loi nº $2010-769$ du 9 juillet 2010, détachant encore un peu plus le droit pénal du droit civil.

68. Cass., crim., 11 juin 1992, n $91-86.346$, Dalloz, 1993, Jur., p. 117, note M.-L. Rassat; Dalloz, 1993, Somm., p. 13, obs. G. Azibert; La semaine juridique, éd. G, 1993, II, 22043 et I, 3639, note T. Garé; Revue de sciences criminelles, 1993, p. 330, obs. G. Levasseur; La semaine juridique, éd. G, 1993, I, 3641, n 3, obs. M. Véron. Voir aussi Cour EDH, C. R. et S. W. c. Royaume-Uni, 22 novembre 1995, série A, n 335-C et 335-B, Revue de sciences criminelles, 1996, p. 473, obs. R. Koering-Joulin; P. Nuss, «La condamnation par la Cour européenne des droits de l'homme du viol entre époux", Gazette du palais, 1997, 2, doctr., p. 945

69. CA Aix-en-Provence, $6^{\mathrm{e}} \mathrm{ch}$. B, 3 mai 2011, La semaine juridique, éd. G, 2011, 1156, obs. L. Pizarro.

70. Pour une décision particulièrement motivée: CA Rouen, chambre correctionnelle, 12 janvier 2011, nº 10/o0490, 40, JurisData, nº 2011-oo2924. En cas de doute sur le consentement, le viol ou les agressions sexuelles sont parfois requalifiés en violences volontaires: voir Y. Zemrak, «La répression des violences conjugales: contribution du juge pénal à la victoire de Lilith sur Ève», Droit de la famille, 2008 , étude n 18.

71. Code pénal, art. 222-18-3.

72. Elle est d'ailleurs insérée dans l'alinéa 2 de l'article 222-14 relatif aux violences sur mineurs.

73. Puni de trente ans de réclusion dans le premier cas et de vingt ans dans le second.

74. Puni de dix ans d'emprisonnement et de $150000 €$ d'amende lorsque les faits ont entraîné une incapacité totale de travail de plus de huit jours et de cinq ans d'emprisonnement et de $75000 €$ d'amende dans les autres cas.

75. Code pénal, art. 222-33-2-1. L'infraction s'applique aussi aux «ex» (même article, alinéa 2). 
moral au travail ${ }^{76}$, cette infraction s'en détache toutefois nettement par le fait qu'elle n'est pas formelle mais matérielle: une altération de la santé physique ou mentale de la victime doit être caractérisée ${ }^{77}$. Cela explique la gradation des peines en fonction de la gravité de l'atteinte ${ }^{78}$. Ces deux qualifications nouvelles peuvent a priori sembler redondantes, dans la mesure où le harcèlement suppose la répétition, donc l'habitude. Mais elles ne le sont pas nécessairement, la première visant les violences physiques, étant intégrée dans la section relative aux atteintes à l'intégrité de la personne, la seconde les violences morales, insérée dans une section distincte relative au harcèlement moral. Toutefois, les cartes se brouillent dès lors que la loi a expressément assimilé les violences physiques et psychologiques ${ }^{79} \ldots$

Enfin, la loi du 9 juillet 2010 tend aussi à lutter contre une autre forme de violence, qui se déroule en amont: le mariage forcé. La loi du 4 avril 2006 avait déjà, en ce sens, hissé l'âge minimal du mariage à 18 ans, la majorité civile. La loi de 2010 va beaucoup plus loin. Tout d'abord, elle crée une nouvelle circonstance aggravante, fondée sur le mobile: contraindre à contracter mariage (ou une union de même nature), ou en raison du refus de contracter mariage (ou une union de même nature). Cette circonstance aggravante est prévue pour le meurtre, les actes de tortures et de barbarie, les violences volontaires de toute gravité ${ }^{80}$. Ensuite, prenant en compte que les mariages forcés sont surtout contractés à l'étranger, la loi pose une dérogation aux règles de compétence territoriale. En principe, la loi pénale française ne peut s'appliquer que si l'infraction est commise en France, ou, sous certaines conditions, si la victime est de nationalité française ${ }^{81}$. Désormais, elle est aussi compétente lorsque la victime effective ou potentielle d'un mariage forcé quoiqu'étrangère réside «habituellement sur le territoire français », sans exiger, pour les délits, une plainte de la victime ${ }^{82}$. Pour compléter le dispositif, l'article 34 de la loi (non codifié) prévoit que:

[1]es autorités consulaires françaises prennent les mesures adaptées pour assurer, avec leur consentement, le retour sur le territoire français des personnes de nationalité française ou qui résident habituellement de manière régulière sur le territoire français lorsque ces personnes ont été victimes à l'étranger de violences volontaires ou d'agressions sexuelles commises dans le cadre d'un mariage forcé ou en raison de leur refus de se soumettre à un mariage forcé ${ }^{83}$.

Certaines de ces dispositions protectrices du couple ne sont pas en parfaite cohérence avec celles qui protègent la famille. L'exemple le plus flagrant tient au rapprochement des immunités jouant au profit de l'auteur d'une infraction contre les biens de son conjoint et de l'aggravation de la répression des violences lorsqu'elles sont commises par ce même conjoint. Ainsi, l'extorsion est définie comme:

[...] le fait d'obtenir par violence, menace de violences ou contrainte soit une signature, un engagement ou une renonciation, soit la révélation d'un secret, soit la remise de fonds, de valeurs ou d'un bien quelconque ${ }^{84}$.

La violence, physique ou morale, est inhérente à l'extorsion. Or, ce délit bénéficie de la même immunité que celle prévue pour le vol, c'est-à-dire qu'aucune poursuite pénale n'est possible lorsque l'extorsion est commise au préjudice de son conjoint, sauf lorsque les époux sont séparés de corps ou autorisés à résider séparément et sauf si l'infraction porte sur des biens indispensables à la vie quotidienne. On en arrive à une situation étrange: les violences « crapuleuses » à l'égard du conjoint bénéficient d'une faveur légale peu compréhensible ${ }^{85} \ldots$

Sanctionner le coupable ne suffit pas. Il faut aussi veiller à faire cesser l'infraction et prévenir son renouvellement. À cette fin, la loi pénale organise aussi la séparation du couple.

\section{B. La protection des personnes par la séparation du couple}

La victime de violences ou de maltraitances conjugales peut évidemment demander le divorce, rompre le pacs ou quitter le domicile commun. Mais les choses ne sont pas toujours aussi simples. La séparation peut se heurter à des obstacles juridiques ou matériels, et, de plus, le danger ne disparaît pas du seul fait de la rupture du couple, ni même avec le déclenchement de l'action publique ou la condamnation pénale si cette dernière n'est pas adaptée à la situation. C'est pourquoi le législateur a jugé bon d'organiser et parfois d'imposer la séparation du couple.

76. Code pénal, art. 222-33-2.

77. Pour le délit de harcèlement au travail, une dégradation des conditions de travail doit être constatée, mais il suffit qu'elle soit «susceptible», notamment, d'altérer la santé physique ou mentale.

78. Trois ans d'emprisonnement et $45000 €$ d'amende lorsque les faits ont causé une incapacité totale de travail inférieure ou égale à huit jours ou n'ont entraîné aucune incapacité de travail, et cinq ans d'emprisonnement et $75000 €$ d'amende lorsqu'ils ont causé une incapacité totale de travail supérieure à huit jours.

79. Code pénal, art. 222-14-3.

80. Code pénal, art. $221-4,10^{\circ}$; art. 222-3, $6^{\circ}$ bis; art. 222-8, $6^{\circ}$ bis; art. 222-10, $6^{\circ}$ bis; art. 222-12, $6^{\circ}$ bis; art. $222-13,6^{\circ}$ bis.

81. Code pénal, art. 113-7 et 113-8.

82. Code pénal, art. 221-5-4 (meurtre), art. 222-6-3 (actes de tortures) et art. 222-16-3 (violences).

83. Loi $\mathrm{n}^{\circ} 2010-769$ du 9 juillet 2010 relative aux violences faites spécifiquement aux femmes, aux violences au sein des couples et aux incidences de ces dernières sur les enfants, art. 34

84. Code pénal, art. 312-1.

85. La même remarque pourrait être faite pour le vol assorti de la circonstance aggravante de violences, sous réserve qu'il est alors envisageable de scinder les faits et de poursuivre uniquement du chef de violences. Pour une solution contraire, voir C. de Jacobet de Nombel, Théorie générale des circonstances aggravantes, Paris, Dalloz (Nouvelle bibliothèque de thèses), 2006, vol. 55, p. 374 . 
La loi du 26 mai 2004 avait créé le "référé violences " ${ }^{86}$, auquel la loi du 9 juillet 2010 a substitué l'ordonnance de protection. Cette ordonnance permet au juge aux affaires familiales, "lorsque les violences exercées au sein du couple ou par un ancien conjoint, concubin, ou partenaire, mettent en danger la personne qui en est victime ou un ou plusieurs enfants ", d'imposer, notamment, des résidences séparées et d'interdire de rencontrer ou d'entrer en relation avec certaines personnes ${ }^{87}$. La victime doit d'ailleurs être informée, lors du dépôt de sa plainte auprès de la police, de son droit de solliciter cette ordonnance ${ }^{88}$. Le juge aux affaires familiales devient un quasi-juge pénal, devant apprécier s'il existe des « raisons sérieuses de considérer comme vraisemblables» les violences et le danger allégués ${ }^{89}$. L'ordonnance de protection s'inspire en effet largement de dispositions déjà prévues en matière pénale.

L'interdiction de paraître dans certains lieux ou de rencontrer certaines personnes, notamment les victimes de l'infraction, sont des obligations classiques du sursis avec mise à l'épreuve et du contrôle judiciaire ${ }^{90}$. La loi $\mathrm{n}^{\circ} 2005^{-}$ 1549 du 12 décembre 2005, relative au traitement de la récidive, s'en est inspirée pour créer une mesure spécifique, propre à assurer la sécurité de la victime de violences commises par son conjoint ou son concubin ${ }^{91}$, mesure qui fut rapidement étendue, par la loi du 4 avril 2006, au partenaire lié par un pacs et aux «ex», et à toutes les phases de la procédure. Il s'agit de l'obligation de résider hors du domicile ou de la résidence du couple et, le cas échéant, de s'abstenir de paraître dans ce domicile ou cette résidence ou aux abords immédiats de celui-ci. Lorsque l'infraction a été commise par l'ancien conjoint, partenaire ou concubin, le domicile concerné est celui de la victime. Cette mesure présente la particularité de pouvoir intervenir à divers stades de la procédure, avant, pendant ou à l'issue des poursuites, ou encore dans le cadre de l'exécution de la peine: elle peut être demandée par le procureur de la République à l'occasion d'un classement sous condition ${ }^{92}$ ou proposée dans le cadre d'une composition pénale ${ }^{93}$; elle peut être une obligation du contrôle judiciaire en cours d'instruction ${ }^{94}$; elle peut assortir un sursis avec mise à l'épreuve prononcé par la juridiction de jugement ${ }^{95}$ et diverses modalités d'exécution de l'emprisonnement décidées par le juge de l'application des peines ${ }^{96}$; elle peut enfin constituer une mesure post-carcérale imposée lors de la libération anticipée ${ }^{97}$. Le non-respect de ces obligations sera diversement sanctionné selon le cadre dans lequel elles s'inscrivent: déclenchement de l'action publique s'il s'agissait d'une alternative aux poursuites, placement en détention provisoire s'il s'agissait d'une obligation du contrôle judiciaire, révocation du sursis avec mise à l'épreuve, réincarcération en cas de libération anticipée.

Afin d'assurer le contrôle de ces obligations, le législateur a complété le dispositif en instaurant ce que l'on pourrait appeler des «diminutifs de garde à vue», tant ils s'en inspirent dans leurs conditions et modalités d'application. Le premier, issu de la loi $\mathrm{n}^{\circ} 2010-242 \mathrm{du} 10$ mars 2010 tendant à amoindrir le risque de récidive criminelle, est prévu à l'encontre de condamnés placés sous le contrôle du juge de l'application des peines: sursitaires, condamnés bénéficiant de mesure d'aménagement de leur peine ou d'une libération anticipée ${ }^{98}$. Le second, issu de la loi du 9 juillet 2010, concerne les personnes mises en examen placées sous contrôle judiciaire par le juge d'instruction ou le juge des libertés et de la détention ${ }^{99}$. Ces « diminutifs de garde à vue» permettent aux services de police ou de gendarmerie, d'office ou sur instruction du juge, d'appréhender et de retenir durant vingt-quatre heures une personne à l'encontre de laquelle il existe «une ou plusieurs raisons plausibles de soupçonner» qu'elle a manqué aux obligations pesant sur elle de s'abstenir de rencontrer ou d'entrer en relation avec la victime ou de paraitre dans certains lieux ${ }^{100}$.

86. Code civil, art. 220-1, al. 3 anc

87. Code civil, art. 515-9 sq. Voir E. Bazin, «les nouveaux pouvoirs du JAF en matière de violences au sein des couples», La semaine juridique, éd. G, 2010, I, 957 ; F. Defferrard, «La suspicion légitime contre les violences au sein des couples - ou le nouveau référé protection», Droit pénal, 2010, étude $\mathrm{n}^{\circ} 27$.

88. Code de procédure pénale, art. 53-1 et 75 .

89. Code civil, art. 515-11. Par ailleurs, le non-respect d'une ordonnance de protection délivrée par le juge aux affaires familiales constitue un délit (Code pénal, art. 227-4-2).

90. Pour le sursis avec mise à l'épreuve, voir Code de procédure pénale, art. 739 et R. 39 anc., aujourd'hui insérés dans le Code pénal (art. 132-45, spéc. $9^{\circ}$ et $13^{\circ}$ ). Pour le contrôle judiciaire: Code de procédure pénale, art. 138 , spéc. $3^{\circ}$ et $9^{\circ}$. Ces mesures sont aujourd'hui possibles dans le cadre des alternatives aux poursuites et de l'application des peines (Code de procédure pénale, art. $41-2,9^{\circ}$ et $10^{\circ}$ et art. 712-16-2).

91. Applicable aussi aux violences commises sur les enfants.

92. Code de procédure pénale, art. 41-1, $6^{\circ}$.

93. Code de procédure pénale, art. $41-2,14^{\circ}$.

94. Code de procédure pénale, art. $138,17^{\circ}$.

95. Code pénal, art. $132-45,19^{\circ}$.

96. Fractionnement ou suspension de la peine (Code de procédure pénale, art. 720-1); placement à l'extérieur, semi-liberté, permission de sortie (Code de procédure pénale, art. 723-4). Ces articles renvoient à l'article 132-45 du Code pénal.

97. Surveillance judiciaire (Code de procédure pénale, art. 723-30); libération conditionnelle (Code de procédure pénale, art. 731); suivi socio-judiciaire (Code pénal, art. 131-36-2). Ces articles renvoient à l'article 132-45 du Code pénal.

98. Code de procédure pénale, art. 712-16-3. Voir A.-G. Robert, Chronique législative, Revue de sciences criminelles, 2010, p. 929.

99. Code de procédure pénale, art. 141-4.

100. La personne retenue bénéficie de garanties inspirées de celles de la garde à vue: information immédiate du juge d'instruction ou du juge de l'application des peines, droit de faire prévenir une personne, droit à un examen médical, droit à un entretien d'une demi-heure avec un avocat. Le droit à l'assistance d'un avocat durant l'audition n'est en revanche pas prévu. 
La retenue du suspect a pour objet de vérifier sa situation et de l'entendre sur la violation de ses obligations. L'intérêt de la mesure tient, du côté des victimes, au fait que l'arrestation et l'audition qui suivra peut être décidée d'office, sans passage préalable par le juge, qui doit toutefois être informé dès le début de la mesure. Un signalement au commissariat peut ainsi suffire, ce qui permet d'agir rapidement.
Ainsi, se construit petit à petit un droit pénal du couple, situé au confluent du droit pénal de la famille et du droit pénal des personnes. Constitué de dispositions éparses ne poursuivant pas toutes les mêmes objectifs (protéger le couple ou protéger ses membres), il manque parfois de cohérence. Il est peut-être temps que le législateur envisage d'harmoniser tout cela. 